Ann. Biol. anim. Bioch. Biophys., Ig66, 6 (4), 529-532.

\title{
PRODUCTION DES HÉMOLYSINES STAPHYLOCOCGIQUES DELTA ET GAMMA
}

\author{
M. PLOMmeT et C. BOUILLANNE \\ Station de Pathologie de la Reproduction, \\ Centre de Recherches vétérinaires et zootechniques, 37 - Nouzilly: \\ Station centrale de Recherches laitières, \\ Centre national de Recherches zootechniques, 78 - Jouy-en-Josas.
}

La production régulière d'une toxine d'un titre élevé présente toujours toute une série de difficultés pour les laboratoires non spécialisés. Pendant des années, nous avons produit la toxine staphylococcique delta par culture sur feuilles de cellophane selon Marks et VAughan (I950) et Jackson et LitThLe (I95 $5^{8}$ b). Les titres sont le plus souvent convenables et le produit exempt des fractions non dialysables du milieu. Malgré différentes améliorations concernant la composition du milieu, cette fabrication reste fastidieuse et limitée en volume. YoshIDA (I963) réussit la production en milieu liquide, sous le volume de I litre, en utilisant le milieu CCY de GLADSTONE et VAN HEYNINGEN (I957). C'est cette méthode que nous avons utilisée et améliorée pour la production des toxines delta et gamma, et avec laquelle nous avons obtenu des résultats satisfaisants sur respectivement 23 et 3 I fabrications.

La distinction entre les toxines staphylococciques est actuellement basée : $I^{\circ}$ sur leur activité hémolytique envers les hématies de mouton, d'homme, de lapin et de cheval ; $2^{\circ}$ sur la thermorésistance à $60^{\circ}$ pendant ro mn; $3^{\circ}$ sur $1^{\prime}$ action neutralisante de la cystéine et de l'acide ascorbique (ELEK et LEVY, I950; JACKSON et I,ITTLE, I958 a; JACKSON, I962). La toxine $\delta$ à laquelle nous nous référons ici est thermostable, active sur les hématies des quatre espèces, en particulier sur les hématies d'homme et de cheval et agit en synergie avec la toxine $\beta$ sur les globules de mouton en boîte de gélose, par un phénomène identique au phénomène de Camp (ELEK et LÉVY, I954). La plupart de nos essais ont été faits avec la souche $B .22$ de ELEK et LÉVY (I954) laquelle produit surtout de la toxine $\delta$ et un peu de toxine $\gamma$. Yoshida utilisait la souche Foggie de Gradstone, qui produit aussi parfois de la toxine $\beta$, ce qui risque de perturber les titrages. La toxine $\gamma$ est thermolabile, active sur les globules humains, inactives sur les globules de cheval et ne donne pas la réaction de Camp. JACKSON et LITTLE (I95 8 ) ont décrit une fraction $\delta$ thermolabile, retenue dans certaines conditions sur filtre de verre ultra-fin. La distinction 
entre cette fraction et la toxine $\gamma$ n'est pas claire. Dans nos essais, la toxine $\gamma$ a été obtenue par la souche $5 R$ de Smith (1962) qui ne donne pas de toxine $\delta$.

Les titrages des toxines $\gamma$ et $\delta$ ont été faits par la technique de JAckson et LITTLE (I958 a), avec une incubation de I 5 minutes à $37^{\circ} \mathrm{C}$. L'unité est définie comme la quantité produisant l'hémolyse à 50 p. Ioo d'une suspension à I p. Ioo d'hématies humaines sous le volume total de $0,7 \mathrm{ml}$. L'unité définie par Yosmida (I963) vaut I,42 unité selon JACKSON. I’unité définie par KAYSER et RAYNAUD (I965), dans un système différent, est approximativement équivalente à $0,3 \mathrm{U}$ selon JAckson. Les titrages de $\gamma$ et $\delta$ sont approximativement additifs, ce qui permet de calculer $\gamma$ par différence entre les titres avant et après chauffage. La courbe de l'hémolyse en fonction de la concentration de toxine est une droite sur papier log-probit (KAYSER et RAynACD, r965) ; l'angle sur la verticale est de $40^{\circ}$ avec la toxine $\delta$ et de $20^{\circ}$ avec la toxine $\gamma$. Avec les mélanges de toxine on obtient des pentes intermédiaires.

Les souches productrices sont conservées sur culot de gélose, au réfrigérateur, et périodiquement purifiées sur gélose au sang humain. La simple sélection des colonies entourées des zones d'hémolyse les plus larges assure une bonne régularité de la production. La souche est repiquée de la gélose dans un erlenmeyer de $50 \mathrm{~m} 1$ contenant Io $\mathrm{ml}$ de milieu $\mathrm{CCY}$, puis incubée au bain-marie à $37^{\circ} \mathrm{C}$ avec agitation rotative vigoureuse par agitateur magnétique pendant environ 3 heures. I,a totalité de cette culture est alors portée dans un erlenmeyer de $250 \mathrm{ml}$ contenant $50 \mathrm{~m} 1$ de milieu, incubée à son tour dans les mêmes conditions pendant 4 heures. Après ce délai, la culture renferme environ $5 \cdot \mathrm{IO}^{9}$ bactéries par $\mathrm{ml}$; elle est transférée en totalité dans un flacon à toxine (fig. I) maintenu dans un bain-marie à $37^{\circ} \mathrm{C}$ conte-

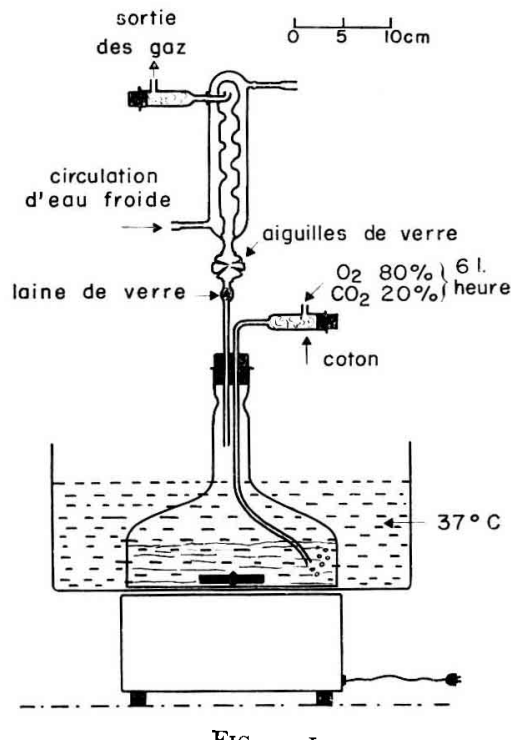

Fig. - I

nant I 1 de milieu et un produit antimousse. La culture est aérée par barbotage d'un mélange de 80 p. Ioo d' $\mathrm{O}_{2}$ et de 20 p. Ioo de $\mathrm{CO}_{2}$, dont le débit approximatif est de $61 / \mathrm{h}$, et agitée d'un mouvement circulaire par un agitateur magnétique. 
Un condenseur et un réfrigérant assurent l'évacuation des gaz sans contamination du filtre de coton. La vitesse d'agitation et la quantité d'antimousse sont adaptés à chaque toxine et chaque souche. Pour la toxine $\delta$, avec la souche $B 22$, il faut une agitation très vigoureuse, au maximum de l'agitateur, soit environ $900 \mathrm{t} / \mathrm{mn}$, mais il suffit de $0,15 \mathrm{ml}$ de l'antimousse Rhodorsil 426 (Rhône-Poulenc). Ce produit, stérilisé par ébullition, est ajouté après stérilisation du milieu. Pour la toxine $\gamma$ avec la souche $\delta R$, il suffit d'une agitation moyenne créant une petite dépression sur la surface du liquide (environ $700 \mathrm{t} / \mathrm{mn}$ ) mais il faut I $\mathrm{ml}$ d'antimousse.

Les toxines apparaissent vers la Io $^{\mathrm{e}}$ heure, et atteignent leur valeur maximum vers la $16^{\mathrm{e}}$ heure. La toxine $\gamma$ diminue ensuite et finit par disparaître totalement. La quantité de toxine $\gamma$ en fin de culture est grossièrement proportionnelle à la densité de la culture ; on obtient en général 60 à $80 \cdot 10^{9}$ cellules par ml et un titre de $250 \mathrm{U} / \mathrm{ml}$. La quantité de toxine $\delta$ n'est pas proportionnelle à la densité de la culture, mais paraît surtout dépendre de l'importance de l'aération et de l'agitation. On obtient habituellement $50^{\circ} \cdot \mathrm{IO}^{9}$ cellules et un titre de $300 \mathrm{U} / \mathrm{ml}$. La prolongation de l'incubation n'entraîne pas de modification dans le titre final. Avec les souches produisant les deux toxines la prolongation conduit à une diminution du titre de $\gamma$ et une augmentation relative de $\delta$. Mais avec de telles souches, des facteurs inconnus autres que la durée de la culture interviennent aussi. Notre souche 91-45 a donné, par exemple, en 16 heures, selon les fabrications un titre de $\gamma$ variant, par rapport au titre hémolytique total $(\gamma+\delta)$ de 0 à $70 \mathrm{p}$. roo.

Après incubation, la culture est centrifugée, et le surnageant mis en dialyse. On peut extraire de la toxine $\delta$ du culot microbien lavé par des tampons alcalins (phosphate o,I M, pH I0,5) ou par $\mathrm{NaOH}$ o,or M. La toxine $\delta$ extraite de cette manière est instable en solution neutre.

La dialyse contre eau ou solution de chlorure de sodium provoque généralement. une précipitation avec diminution du titre des toxines $\gamma$ et $\delta$. Nous utilisons une dialyse agitée de $48 \mathrm{~h}$ à $4^{\circ} \mathrm{C}$, contre 4 fois ro volumes de tampon phosphate $0, \mathrm{I} \mathrm{M}$ ( 2 fois) puis $0,005 \mathrm{M}$ ( 2 fois). Dans ces conditions, un faible précipité se forme au passage en $0,005 \mathrm{M}$, mais sans baisse notable du titre. I, a solution en tampon $0,005 \mathrm{M}$ peut être lyophilisée directement en plateau sans baisse du titre, à condition, dans le cas de la toxine $\gamma$, que la température de congélation soit assez basse, environ $-50^{\circ} \mathrm{C}$, pour que $1^{\prime}$ opération soit rapide. La toxine $\delta$ n'est pas sensible à cet effet. Une fois lyophilisées, les toxines se conservent bien avec toutefois une baisse sensible du titre pour la toxine $\gamma$ après 6 mois. Les toxines brutes en solution tampon peuvent être conservées à $4^{\circ} \mathrm{C}$ pendant quelques jours et pendant $2-3$ mois à $-30^{\circ} \mathrm{C}$.

Reçu pour publication en septembre 1966.

\section{SUMMARY}

PRODUCTION OF STAPHYLOCOCCAL DELTA AND GAMMA TOXINS

A lab-method for production of staphylococcal delta and gamma toxins is described. One liter of CCY medium in toxin flask, bubbled with $\mathrm{O}_{2}$ and $\mathrm{CO}_{2}$, seeded with $\log$-phase culture yields evenly high-titer crude toxins. 


\section{RÉFÉRENCES BIBLIOGRAPHIQUES}

ELEK S. D., LEVY E., I950. Distribution of hæmolysins in pathogenic and non-pathogenic staphylococci. J. Palh. Bact., 62, 541-554.

ELEK S. D., LEvy E., I954. The nature of discrepancies between hæmolysins in culture filtrates and plate hemolysin patterns of staphylococci. J. Path. Bact., 68, 3I-40.

Gladstone G. P., Van Heyningen W. E., 1957. Staphylococcal leucocidins. Br. J. exp. Palh., 38, I $23^{-I} 37$.

Jackson A. W., I 962. Staphylococcal $\gamma$-lysin and its differentiation from $\delta$-lysin. VIIIe Congrès Intern. Microbiol, Montréal, p. III.

Jackson A. W., Litrie R. M., 1958 a. Staphylococcal toxins. Factors affecting hemolysis by $\delta$-lysin. Can. J. Microbiol., 4, 435-444.

JACKson A. W., LitTle R. M., 1958 b. Staphylococcal toxins. Partial purification and some properties of $\delta$-lysin. Can. J. Microbiol., 4, 453-46r.

KaYser A., Raynaud M., I965. Étude d'une deuxième hémolysine, distincte de l'hémolyse $\alpha$, présente dans les filtrats de culture de la souche Wood 46 de Staphylococcus aureus (hémolysine G ou $\delta$ ). Ann. Inst. Pasteur, 108, 2I 5-233.

Marks J., Vaughan A. C. T., 1950. Staphylococcal $\delta$-hæmolysin. J. Path. Bact., 62, 597-615.

Smith D. D., 1962. Experimental staphylococcal infection in mice. J. Path. Bact., 84, 359-365.

Yoshida A., I963. Staphylococcal $\delta$-hemolysin. Purification and chemical properties. Biochim., biophys. Acla, 71, 544-553. 